\title{
The Unified Design Methodology for Application Based on XML Schema
}

\author{
Yoon Bang Sung ${ }^{1}$, Mun-Young Choi ${ }^{2}$, and Kyung-Soo Joo ${ }^{3}$ \\ ${ }^{1}$ Hanseo University Computer Center, \\ Hanseo University Computer Center, SeoSan, ChungNam, Korea, 356-706 \\ sybang@hanseo.ac.kr. \\ ${ }^{2}$ Dept. of Computer Science, Graduate School Soonchunhyang Uni. \\ SoonChunHyang Uni., P.O Box 97, Asan, ChungNam, Korea, 336-745 \\ E-mail : griffin@hyejeon.ac.kr. \\ ${ }^{3}$ Dept.of Computer Science and Engineering, College of Engineering SoonChunHyang Uni., \\ P.O Box 97, Asan, ChungNam, Korea, 336-745 \\ gsoojoo@sch.ac.kr
}

\begin{abstract}
In this paper, we introduce a XML modeling methodology to design W3C XML schema using UML and we propose a unified design methodology for relational database schema to store XML data efficiently in relational databases. In the second place, in order to verify objectivity of the unified design methodology. Using Ronald Bourret's method, first we introduce the method of the transformation from XML schema to the object model and second we translate the object model into the relational database schema. Therefore we show the mutual consistency between those consequences, and so can verify the unified design methodology, we proposed in this paper.
\end{abstract}

\section{Introduction}

XML is a markup language for documents that includes the structured information[1]. W3C XML Schema can define more diverse data types than XML DTD, and it has a convenient advantage in using as a diverse application by using the powerful expression. The unified design modeling describes 3WC XML Schema using UML Class and store interchanged data by the modeling in RDB. However, until recently, there has been no effective means to verify objectivity of the unified design modeling. In this study, it describes a unified design methodology in Chapter 2, verification of unified design methodology in Chapter 3, and the conclusion in the last Chapter 4.

\section{Unified Design Methodology Using UML}

The sequential diagram takes on the role to clarify the flow of events from the application program and may convert the use case that is in the form of writing into the picture expressed in massage. In particular, it helps to make easy works when 
making class diagram for us. Then it can be converted to XML modeling and data modeling as shown in the Fig. 1 by the class diagram.

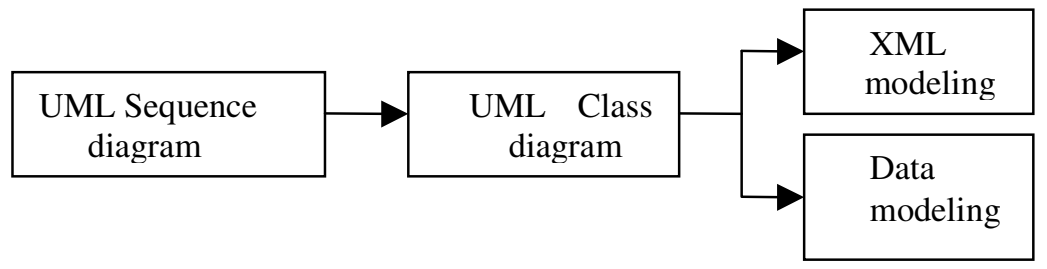

Fig. 1. XML modeling and Data modeling using UML

On Fig.2, the Order and Distributor are aggregate relationships in the relativity. However, It do not illustrate the process, only the information items which participate in the process and the relationships or dependencies between those objects[3].

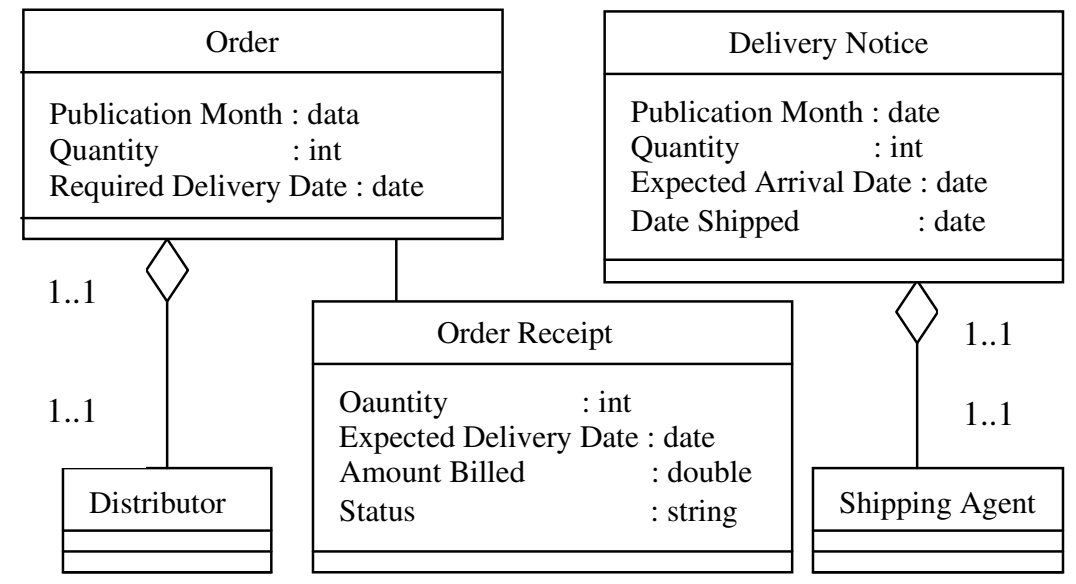

Fig. 2. Class diagram

\subsection{XML Modeling}

The following methods are used to formulate W3C XML Schema from the UML class proposed in the Fig.2[3].

1 Do not allow empty elements or attributes. Instead, ensure that they either have some value or are not present. If an element or attribute is mandatory( such an minOccurs $=" 1$ " or use $=$ "required"), allowing an empty string as the value is questionable. If they are "allowed" to be empty, then it is better to set minOccurs $=" 0$ " or use $=$ "optional", and take their absence to mean "no value" ; if they do exist, force them to have some content. 
2 Since whitespace can increase the size of an instance document significantly, it may be necessary for an application receiving an XML instance document to confirm the file size is under a certain limit.

3 Create limited versions of the base types string, decimal, and integer in order to restrict character set and field size.

4 Make use of the unique element to assert uniqueness of element or attribute values.

5 Use key / keyref elements to associate two locations in a document. Any path in the document can be specified as a named key, such that all paths that are a keyref to that named key must contain a value specified as key.

6 If the schema is in danger of becoming large, consider moving sections into other files using the group reference method. The principle of abstraction applies equally here. These secondary schema files do not need to have even one global element.

7 Wherever we change namespaces in an instance document, use an any element and explicitly specify the namespace and number of child elements permitted at that location, or include an element $r e f="$. . ." element to explicitly name the element in the new namespace.

8 If an element or attribute does not exist, does the application take this to mean there is a default value ? If so, this can explicitly be stated by using the default attribute on the element or attribute declaration.

9 Include the blockDefault="\#all" attribute on the schema element. This will prevent derived types being allowed to substitute in an instance document. This is a security issue, and depends on the application's mechanism for schema validation.

10 Include the finalDefault="\#all" attribure on the schema element. This will prevent any new types deriving from those declared in this schema. This is a much stricter constraint than blockDefault.

\subsection{Example of XML Modeling}

On Fig.2, the 'Order' object and the 'Distributor' object are aggregate relationships in the relativity and its meaning is that the 'Order' object has to have the 'Distributor', and accordingly, the 'Distributor' side is shown to have the multiplicity value of $1 . .1$. On the other hand, the multiplicity value of the 'Order' object being $1 . .1$ means that the 'Distributor' can exist depending on the orders. In order to make XML modeling, modeling with XML Schema by applying the XML modeling method of 1 and 4 for XML Schema for Schema is the same as shown in Fig.3.

$$
\begin{aligned}
& \text { <element name="Distributor"> } \\
& \text { <complexType> } \\
& \text { <attribute name="Distributor" type="ID" use="required"/> } \\
& \text { </complexType> } \\
& \text { </element> }
\end{aligned}
$$

Fig. 3a. XML Schema of Distributor Object(Continute in the next page) 


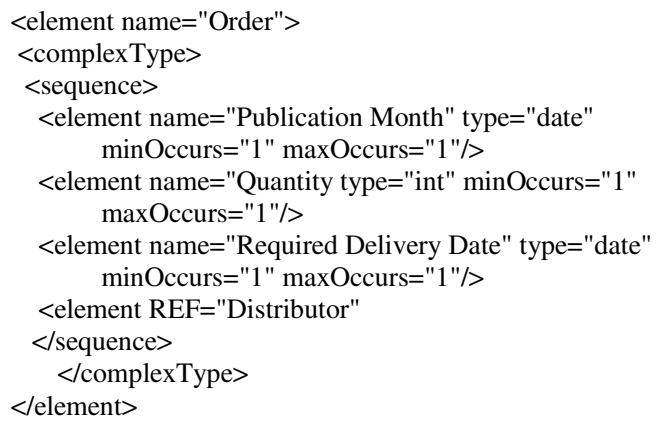

Fig. 3b. XML Schema of Order object(Continue in before page)

On Fig.3, the 'Order' object has 3 child objects and 1 reference object. Also, the 'Distributor' object that refers from the 'Order' object has no child object.

\subsection{Data Modeling}

The conversion methods of class diagram of Fig.2 into RDB Schema is as follows[8].

1 UML class becomes table.

2 UML attribute in class becomes column in table.

3 UML attribute type in class becomes column type in table through type transformation table.

4 IF nullable UML attribute tag, attribute has NULL constraint ; otherwise, NOTNULL constraint.

5 IF UML attribute has initializer, add DEFAULT clause to column.

6 For classes with no generalization(root or independent) and implicit identity, create integer primary key; for oid, addoid tagged columns to PRIMARY KEY constraint; ignore composite aggregation and association classes.

7 For subclasses, add the key of each parent class to the PRIMARY KEY constraint and to a FOREIGN KEY constraint.

8 For association classes, add primary key from each role-playing table to PRIMARY KEY constraint and FOREIGN KEY constraint.

9 IF alternate oid $=<n>$ tag, add columns to UNIQUE constraint.

10 Add CHECK for each explicit constraint.

11 Create FOREIGN KEY columns in referencing table for each $0 . .1,1 . .1$ role in association.

12 Create PRIMARY KEY for composite aggregation with FOREIGN KEY to aggregating table(with CASCADE option), add additional column for PRIMARY KEY.

13 Optimize binary association classes by moving into to-many side table where appropriate.

14 Create tables for many-to-many, ternary associations with no association classes.

15 Create PRIMARY KEY, FOREIGN KEY constraints from keys of role-playing tables in many-to-many, ternary associations. 


\subsection{Example Conversion of RDB Schema}

(1) The 'Distributor' object by Fig.2, It is converted as in Fig.4 with the 'Distributor' table that stores the object type attribute of 'DistributorID' following the characteristics of RDB conversion method 1,2 and 6.

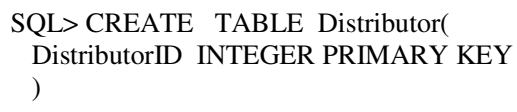

Fig. 4. Distributor Table

(2) The 'Order' object by Fig.2, It stores the 'Order' object type attribute following the characteristics of RDB conversion method number 1, 2 and 6, and the object type of 'DistributorID' is applied with 'Distributor' defined under Fig.4 by conversion method number 11.

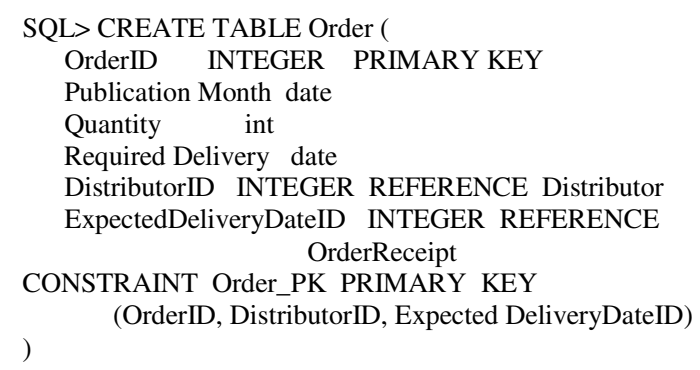

Fig. 5. Order Table

\section{Verification of the Unified Design Methodology Using UML}

In this section, we apply the existing Ronald Bourret's method in order to verify objectivity of the unified design methodology. It transforms XML schema into the object model and transform the object model into relational database schema. Therefore we show the mutual consistency between them and relational database chema according to relational data modeling using UML, and verified objectivity of design methodology.

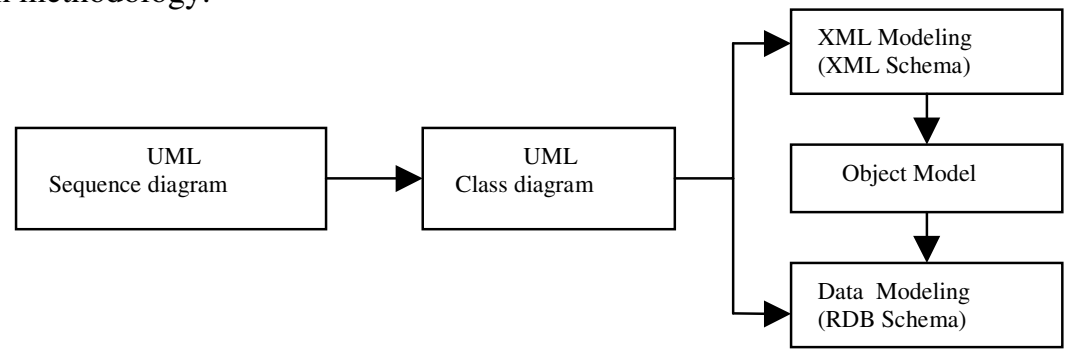

Fig. 6. Verification of design methodology 
Fig.6 describes two different processes that transform XML Schema through the object model into the relational database schema. The first part of the process, transform XML Schema generally known as "XML data binding" to the object model. The second, transform object model known as "object-relational" mapping to the relational database schema[2].

\subsection{Cyclic Graph Expression of XML Schema}

The transformation XML Schema to object model is best understood by viewing an instance of the schema data model as a directed, possibly cyclic graph consisting only of schema, attribute, complex element type, simple element type, attribute group, model group. From this graph, the attribute and the simple element type nodes are transformed into the scalar types. the edges pointing from these nodes are transformed into the properties in these calsses[3]. Fig.7 is an example of XML Schema that transforms Fig.2 into XML modeling.

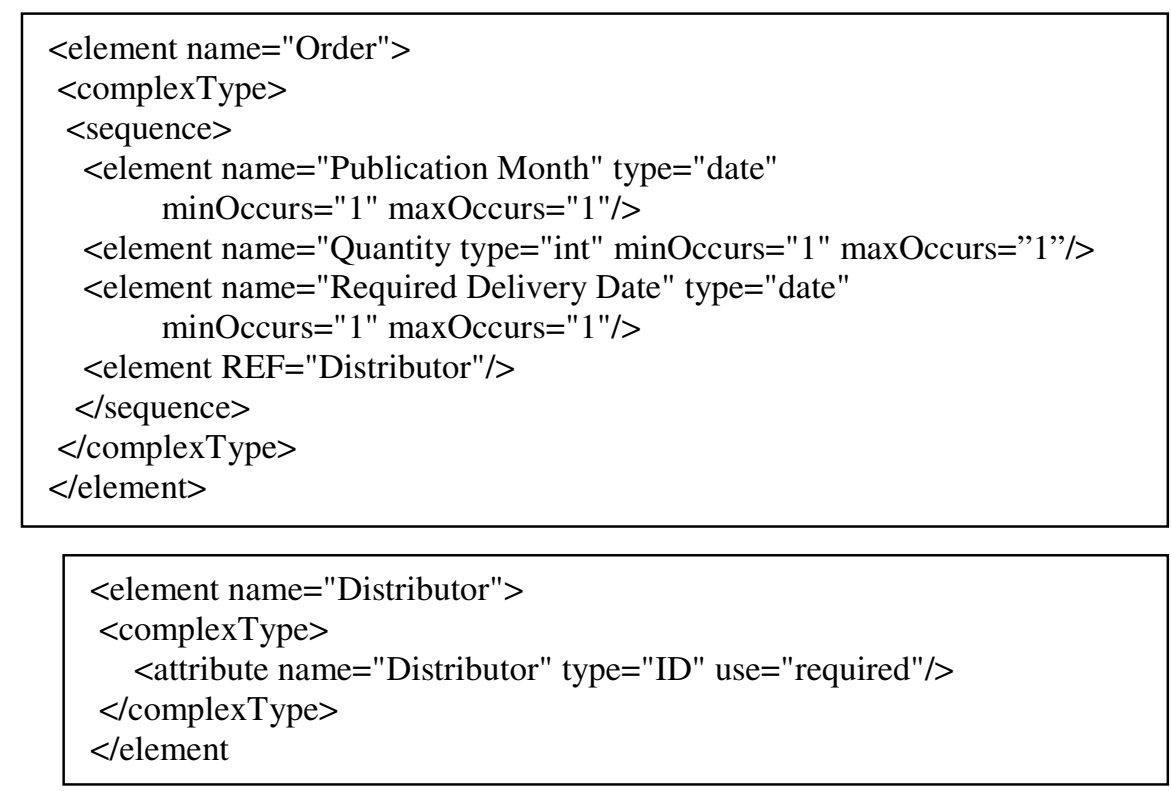

Fig. 7. XML Schema of Distributor(Continue in before page)

\subsection{Element}

$(<$ !ELEMENT $>)$ is the fundamental component in XML DTD and it defines Tag used in XML document. Element in XML Schema is one of the basic components. We use to assign the items that support all each of property in <element $>$, such as an element name, type of Tag content, minimun/maximun number of occurrence of Tag. The element type is classified in two type. Simple element type has no children element 
and complex element type has children element and relationship between elements. Specially, it is useful to define complex type. Simple element type node is generally transformed into scalar data type. Complex element type node is transformed into class. The edges pointing element type node are transformed into property. Fig.8 shows the example that transformed Fig.7 into cyclic graph.

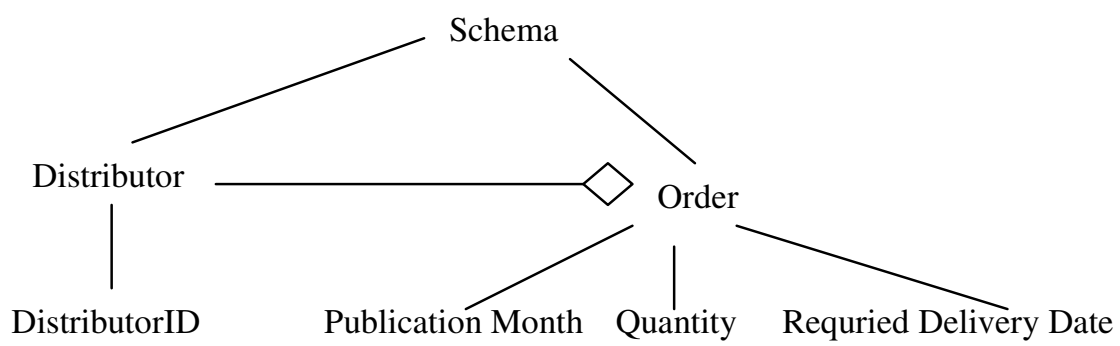

Fig. 8. Cyclic graph of schema component

\subsection{Attribute}

Every element can have an attribute and the attribute has an attribute name and type. The attribute is included in element. The attribute node is transformed into the scalar type and the edges pointing at the attribute node are transformed into their properties. The distributor element node has a property of DistributorID in Fig.9 and becomes a property of Order element node. The Distributor node pointing at Order node with edge $(-\diamond)$ in Fig.8 represented in the object model to mean aggregation between two nodes.

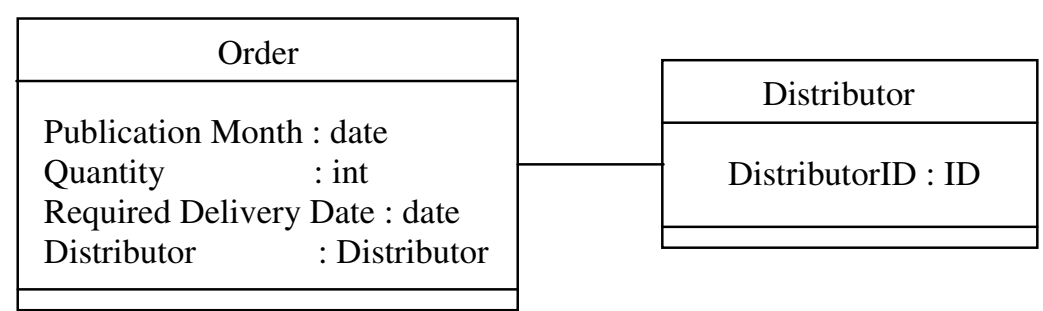

Fig. 9. Order and Distributor object

\subsection{Transforming Object Model into RDB}

We transformed XML Schema Example of Fig.7 in this section 3.1 into the object model. The following method describes how transformed object model are transformed into the relational database schema. 
1 Class : Table. this is known as a class table. an object is represented by a row in a class table.

2 Inheritance : The superclass and subclass are mapped to separate tables with a unique Key/foreign key joining them. The unique key is in the superclass table. An object is represented by a row in each table.

3 Single-valued property with scalar data type : Column in class table. This is known as a property column. The data type determines the set of possible data types of the column. A property is represented by a value in a property column. The property table is joined to the class table with a unique key/foreign key relationship and the unique key is in the class table.

4 Multi-valued(collection) property with scalar data type : Multiple property columns in class table. Each position in the collection is mapped to a specific property column. Property column in a property table. There is one row for each value in the collection. The property table is joined to the class table with a unique key/foreign key relationship and the unique key is in the class table.

5 Single-valued property with union data type : There is one table for each data type in the union and the unique key is in the class table.

6 Single-valued property with class data type : The class containing the property is called the parent class and the class corresponding to the property is called the child class. Unique key/foreign key relationship between the tables of the two classes. The unique key is in the table of the parent class.

7 Multi-valued property with class data type : Unique key/foreign key relationship between the tables of the two classes. The unique key is in the table of the parent class. There is one row in the table of the child class for each property value.

8 Identity constraint: See sections 3.5.

\subsection{Transforming Identity Constraint into RDB}

1 Identity constraint category(key) : The constraint is mapped to a PRIMARY KEY constraint.

2 Identity constraint category(keyref) : The constraint is mapped to a FOREIGN KEY.

3 Identity constraint category(unique) : The constraint is mapped to a UNIQUE constraint.

4 Selector: Must identity a single table. That is, starting from the element type node on which the identity constraint is defined, the XPath expression must lead to a complex element type node.

5 Field : Must identity one or more columns in the table in 4. That is, starting from the node identified in 4, each XPath expression must lead to an edge leading from the node in 4 to simple element type node or an attribute node.

6 Reference key : The selector and fields properties of the referenced key identity constraint are used to determine the table and key columns referenced in a FOREIGN KEY constraint. 


\subsection{Example Conversion of RDB Schema}

(1) The 'Order' object by Fig.9 is converted as in Fig.10 with conversion method that uses RDB conversion method number 1, 4 and RDB identifies constraint number 1 6. It shows the mutual consistency of Fig. 5 and Fig. 10.

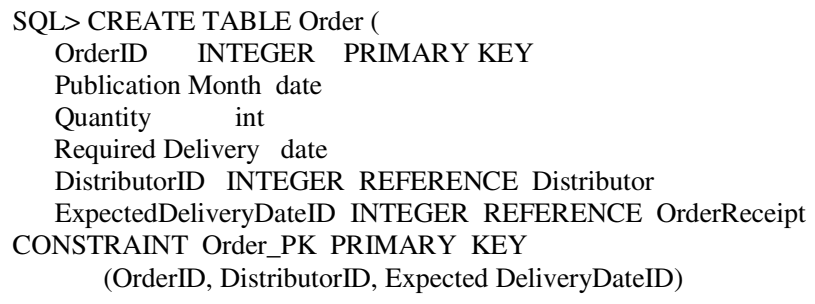

Fig. 10. Order table

(2) The distributor' object by Fig.9 is converted as in Fig.11 with conversion method that uses RDB conversion method number 1, 3. It shows the mutual consistency of Fig.4 and Fig.11.

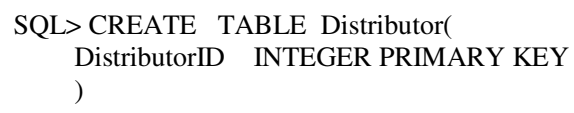

Fig. 11. Distributor table

\section{Conclusion}

In order to store and manage the information mutual exchanged systematically and stably by using XML, there are a variety of studies on XML application and database linkage have been performed on the basis of relational database to this point. However, for there is a limit not to define a variety of data types of DTD in the XML application, there is a difficulty in smoothly linking it to the data base. Furthermore, there is an inherent limit in storing the XML data that has a variety of level structure into the relational database, the aggregation of 2-dimensional table.

In this paper, we proposed modeling methodology to store in relational database for structured information that transformed hierarchical architecture into 2-dimension information using conversion rule. We applied guideline that designed XML modeling and Relational data modeling using UML Class. However, it needs to verify objectivity to repose trust on modeling. In order to verify objectivity, first we transformed XML Schema into relational database and second we transform UML Class into relational database and third we showed the mutual consistency comparing with each other's consequence. For this goal first we represented XML Schema on cyclic graph and transformed edge with node into the object model and transformed object the model into the relational database. We used existing Ronald Bourret's method. 
Acknowledgement. This work was supported by the University IT Research Supporting Program under the Ministry of Information Communication of Korea.

\section{References}

[1] What is XML ?, http://www.xml.com/pub/a/98/10/guide1.html\#AEN58.

[2] Mapping W3C Schemas to Object Schemas to Relational Schemas, http://www.rpbourret.com/xml/SchemaMap.htm.

[3] Duckett Jon, Ozu Nik, Williams Kevin, Mohr Stephen, Cagle Jurt, Griffin Oliver, Norton Francis, Stokes-Rees Ian, and Tennison Jeni. Professional Xml Schemas, Wrox Pr Inc, 2001 .

[4] Florescu, D., Kossmann, D.: Storing and Querying XML Data using an RDBMS. Data Engineering 22:3 (1999), 27-34.

[5] XML Modeling, http://www.xmlmodeling.com

[6] Modeling XML vocabularies with UML, http://www.xml.com/pub/a/2001/09/19/uml.html, 09/19/2001.

[7] Bang Sung-yoon, Joo Kyung-soo, "XML Application Design Methodology using Model of UML Class", Korear of Institute of CALS/EC, The Journal of Korean Institute of CALS/EC, Vol.7, No.1, pp.154-166, 2002.

[8] Bang Sung-yoon, Joo Kyung-soo, "A Unified Design Methodology using UML for XML Application based on Database", Korean Society for Information Management, Journal of the Korean Society for Information Management Vol.19, No.2, pp.50-67, 2002. 